\title{
Rastreamento da pressáo arterial em uma cidade do interior de Minas Gerais: um relato de experiência
}

\author{
Blood pressure screening in a countryside city in the State of Minas Gerais, \\ Brazil: an experience report
}

\section{RESUMO}

Este relato tem como objetivo descrever a experiência e a importância de um projeto de extensão desenvolvido por alunos e professores da Universidade Federal de Uberlândia em parceira com o Programa de Rastreamento da Pressão Arterial no Brasil em outubro de 2016. Relata como foram desenvolvidas as atividades de rastreamento, orientação e educação em saúde cardiovascular para a população local. Descreve o local da intervenção e quais instrumentos utilizados para a realização das açôes para rastreamento dos valores pressóricos além dos materiais para intervenção e educação em saúde. Como resultados, obtivemos os valores da pressão arterial da população e suas características sociodemográficas e clínicas. Concluímos a importância da continuidade de realizaçáo e apoio em pesquisas para o desenvolvimento de diretrizes para o rastreamento da pressão arterial com intuito de minimizar seus agravos e complicações.

Palavras-chave: Doenças cardiovasculares. Pressão arterial. Educação em saúde.

\section{ABSTRACT}

This report describes the experience and importance of an extension project developed by students and professors of the Federal University of Uberlandia-Brazil in partnership with the Program of Tracking of Blood Pressure in Brazil in October, 2016. It reports on the activities of tracking, orientation and education in cardiovascular health for the local population. It describes the location of the intervention and which instruments were used to perform the actions to monitor pressure values in addition to materials for health intervention and education. As results, we obtained the values of the arterial pressure of the population and their sociodemographic and clinical characteristics. We conclude on the importance of the continuity of accomplishment and support in research for the development of guidelines for tracking of blood pressure in order to minimize its complications.
Willian Alves Fonseca

Graduando em Enfermagem (Bacharelado e Licenciatura) pela Faculdade de Medicina da Universidade Federal de Uberlândia, Minas Gerais, Brasil (willianwaf@yahoo.com.br).

Patrícia Magnabosco

Doutora em Enfermagem pela Universidade de São Paulo, Brasil; professora da Faculdade de Medicina da Universidade Federal de Uberlândia, Minas Gerais, Brasil (patriciamagnabosco@hotmail.com)

Omar Pereira de Almeida Neto

Doutor em Atençấo à Saúde pela Universidade Federal do Triângulo Mineiro, Minas Gerais, Brasil; professor na Faculdade de Medicina da Universidade Federal de Uberlândia, Minas Gerais, Brasil (omarpneto@ hotmail.com).

Valéria Nasser Figueiredo

Pós-Doutorado em Ciências Médicas na Universidade Estadual de Campinas, São Paulo, Brasil; professora da Faculdade de Medicina da Universidade Federal de Uberlândia, Minas Gerais, Brasil (valeria.n.figueiredo@gmail.com). 
Keywords: Cardiovascular diseases. Blood pressure. Health education.

\section{INTRODUÇÃO}

Dentre as doenças crônicas não transmissíveis, a Hipertensão Arterial Sistêmica (HAS) destaca-se como o principal fator de risco para doenças cardiovasculares, com uma prevalência na população adulta segundo inquérito nacional de $30 \%$, considerando os valores da pressão arterial, segundo a VII Diretrizes Brasileiras de Hipertensão Arterial $\geq 140 / 90 \mathrm{mmHg}$ (JARDIM et al., 2016; LOBO et al., 2017). Por estar associada diretamente com a autopercepção de saúde ruim dos indivíduos, indica o grau de qualidade de vida da populaçáo, gerando impacto negativo nas atividades de lazer e trabalho, além do alto custo econômico sendo considerado um grave problema de saúde pública devido a seu caráter crônico e incapacitante (ARRUDA et al., 2015).

Segundo o Ministério da Saúde (2010), o rastreamento da HAS é uma ferramenta que não implica um diagnóstico fechado, pois geralmente são exames que selecionam as pessoas com maior probabilidade de apresentar a doença em questáo. Desta forma, a acurácia das medidas da pressão arterial torna-se a base necessária para avaliação da HAS (BEEVERS; LIP; O’BRIEN, 2001). A importância desse rastreamento consiste na prevençáo de agravos à saúde, pois quando não diagnosticada e tratada previamente, a HAS evolui gerando lesóes de órgão alvo como o coração, rins, vasos sanguíneos e o encéfalo, com consequentes agravos temporários ou permanentes (TOLEDO; RODRIGUES; CHIESA, 2007).

É de fundamental importância o desenvolvimento de estudos epidemiológicos de base populacional para se conhecer a distribuição da exposição e do adoecimento por hipertensão no país e os fatores e condiçôes que influenciam a dinâmica desses padrôes de risco na comunidade (PASSOS; ASSIS; BARRETO, 2006). Ao considerar a importância da estratégia de rastreamento dos valores da $\mathrm{PA}$, como importante meio de atenuar as complicaçóes cardiovasculares, ensaios clínicos controlados constataram a efetividade do controle intensivo da HAS (ESTÁCIO et al., 2000; SCHRIER et al., 2002). 
Com a finalidade da redução desses agravos, torna-se essencial enfatizar a realizaçáo de um projeto de rastreamento da HAS que permita identificar e encaminhar os participantes na busca de controle dessa doença e de como prevenir futuras comorbidades. Assim, a Liga Mundial de Hipertensáo, uma organização beneficente sem fins lucrativos, aliada à Sociedade Internacional de Hipertensão e à Organização Mundial da Saúde, sob a liderança de Dr. Norm Campbell, um proeminente pesquisador no campo da HAS, estabeleceu, no ano de 2014, um Comitê para apoiar o desenvolvimento do rastreamento da hipertensão arterial sistêmica nos países emergentes. O Comitê tinha como objetivo a criação de diretrizes para rastreamento da hipertensão, desenvolver materiais, programas de treinamento para promover a detecção, o controle e prevenção da HAS na população.

Tendo como foco os países emergentes, deu-se início a um projeto piloto no Brasil, proposto pela Profa. Dra. Eugenia Velludo Veiga (Universidade de São Paulo). Foram desenvolvidas parcerias junto ao Grupo de Pesquisa da Oficina de Projetos em Esfigmomanometria, Departamento de Enfermagem Geral e Especializada da Escola de Enfermagem de Ribeirão Preto da Universidade de São Paulo.

O estudo iniciou-se nas cidades de Ribeirão Preto, São Paulo e Franca, no dia 26 de abril de 2015, marcado como "Dia Nacional do Combate a Hipertensão", no qual foi realizado o rastreamento da pressáo arterial e orientaçóes sobre fatores de risco da HAS na comunidade. Paralelamente, foi discutida a possibilidade de ampliar esse tipo de intervenção para outras cidades, tais como Recife, Alfenas, Campinas, Fortaleza, São Paulo, Ribeirão Preto e Goiânia, ocasião em que ocorreria no "Dia do Coraçáo", em 14 de agosto de 2015.

No segundo semestre do ano de 2016, o Programa de Rastreamento da Pressão Arterial no Brasil contou com a parceria da Liga Multiprofissional de Cardiologia (Multicardio) e do Grupo de Pesquisa à Saúde Cardiovascular cadastrado no CNPq, ambos do Curso de Graduação em Enfermagem da Faculdade de Medicina da Universidade Federal de Uberlândia (FAMED/UFU). Os objetivos da liga Multicardio e do grupo de pesquisa envolvem realizar estudo e pesquisas na área de promoção e prevenção à saúde cardiovascular.

Segundo dados do DATASUS (2018), no período de janeiro de 2002 a abril de 2013, na cidade de Uberlândia, foram cadastrados 32.740 
hipertensos no Sistema de Cadastramento e Acompanhamento de Hipertensos e Diabéticos. Os aspectos até aqui mencionados demonstram a problemática da HAS e a importância de investimentos por parte dos órgãos públicos em políticas e estratégias que visem o controle e redução das complicaçôes decorrentes dessa síndrome.

A cidade de Uberlândia-MG é o segundo município mais populoso do estado de Minas Gerais, na Regiáo Sudeste de Brasil. Sua população, segundo estimativas do Instituto Brasileiro de Geografia e Estatística (IBGE), era de 676.613 habitantes em 2017, sendo o município mais populoso da regiáo do Triângulo Mineiro e o segundo mais populoso de Minas Gerais, depois da capital, Belo Horizonte. É, também, o município mais populoso do interior de Minas e o quarto do interior do Brasil, além de ser o $12^{\circ}$ mais populoso do país.

A intervenção foi realizada no Complexo Parque do Sabiá-UberlândiaMG, um local ideal para atividades de lazer e práticas desportivas. O Parque possui uma área de $1.850 .000 \mathrm{~m}^{2}$, com um bosque de $350.000 \mathrm{~m}^{2}$ de área verde, um conjunto de três nascentes que abastecem sete represas e originam um grande lago e sete outros menores; uma praia artificial com 300 metros de extensão; uma pista de caminhada de 5.100 metros de extensão; duas piscinas de água corrente; vários campos de futebol; cinco quadras poliesportivas; uma quadra de areia; um campo de futebol; um completo parque infantil, com mais de 100 brinquedos; conjuntos sanitários; vestiários esportivos; lanchonetes e vários recantos contemplativos, entre outras instalações.

Considerando que a HAS é um sério problema de saúde pública e a sua detecção precoce, bem como seu tratamento, são essenciais para evitar morbidades cardiovasculares de alta mortalidade, este estudo objetiva descrever a experiência e a importância de um projeto de extensão desenvolvido por alunos e professores da Universidade Federal de Uberlândia em parceira com o Programa de Rastreamento da Pressão Arterial no Brasil.

\section{METODOLOGIA}

O tipo do presente estudo foi de abordagem de caráter exploratório de campo, descritivo e transversal, de base populacional. A pesquisa foi aprovada pelo Comitê de Ética em Pesquisa da Universidade Federal 
de Uberlândia, Uberlândia, MG (Número do Parecer: 1.919.425).

A extensão universitária é a interação transformadora entre a Universidade e a comunidade, possibilitando o compartilhamento do conhecimento adquirido por meio de ensino e pesquisa desenvolvidos na instituição com a sociedade em que está inserida, interagindo e transformando a realidade social, além de possibilitar a formação do profissional cidadáo (UFU, 2009). Como prática acadêmica, ela articula o ensino e a pesquisa de forma indissociável e instrumentaliza a relação dialética/prática, por meio de um trabalho inter e transdisciplinar, que favorece uma visão global das questóes sociais, viabilizando a superação das desigualdades sociais existentes (UFU, 2009).

$\mathrm{O}$ atendimento à comunidade foi feito por alunos da Liga Multiprofissional de Cardiologia e do Grupo de Pesquisa à Saúde Cardiovascular da Universidade Federal de Uberlândia, previamente treinados, sob a supervisão de docente vinculado a mesma instituição. A amostra desse estudo foi por conveniência, composta por 68 participantes que compareceram espontaneamente ao Complexo Parque do Sabiá, Uberlândia-MG, em 1º de outubro de 2016.

Utilizamos como critérios de inclusão indivíduos de ambos os sexos que concordaram em participar do estudo e que assinaram o Termo de Consentimento Livre Esclarecido, após claramente entendê-lo e critério de exclusão a população menor de 18 anos de idade. E, durante a realização do evento, os participantes foram inquiridos sobre idade, sexo, cor, peso, altura e medida indireta da pressão arterial (PA).

$\mathrm{Na}$ aferição da medida indireta da PA, primeiramente utilizamos uma fita métrica não distensível para medir a circunferência do braço dos participantes, para, a partir dessa mensuração, escolher a braçadeira adequada para cada indivíduo. Os equipamentos utilizados para a medida da PA foram aparelhos portáteis, semiautomáticos e/ou automáticos e manguitos de diferentes tamanhos. As verificaçôes da PA foram realizadas com o indivíduo em posição sentada, em repouso por 5 minutos.

Todos os participantes foram submetidos a três aferiçôes da PA em repouso, com intervalo de um minuto entre elas. Para fins do estudo, foi considerada a média dos valores das duas últimas medidas. A 
partir do resultado, foi classificada a PA obtida de acordo com as orientaçóes estabelecidas na VI Diretrizes Brasileiras de Hipertensão Arterial, 2010.

Foram considerados hipertensos os participantes com pressão sistólica $>140 \mathrm{mmHg}$ e/ou pressão diastólica > $90 \mathrm{mmHg}$, levando-se em conta a segunda medida de PA e ou na abordagem onde houve o relato de uso de anti-hipertensivos.

As orientaçóes após a aferição da PA dos participantes foram realizadas por meio de diálogo educativo concomitante à entrega de fôlderes explicativos, contendo informaçôes sobre as consequências da hipertensão, o controle dos valores pressóricos, o uso de alimentação com pouco sal e o controle para os hipertensos do uso continuo da medicação com o acompanhamento médico frequente.

\section{RESULTADOS E DISCUSSÃO}

Munidos desse conhecimento teórico, rastreamos os valores pressóricos de 68 indivíduos, sendo $50 \%$ homens e $50 \%$ mulheres com média de idade de $58 \pm 16$ anos, índice de massa corpórea (IMC) foi de $27 \pm 4$ $\mathrm{kg} / \mathrm{m}^{2}$. Dentre eles, $50 \%$ confirmaram uso contínuo ou prévio de um ou mais medicamentos anti-hipertensivos, sendo considerados como população hipertensa. Esse método de rastreamento é considerado válido e útil para a vigilância dessa patologia, uma vez que estudos atestam uma boa validade do autorrelato de hipertensão arterial sistêmica, com sensibilidade que varia de 71,1\% a 84,3\% e especificidade de $80,5 \%$ a $87,5 \%$. Além disso, trata-se de uma estratégia rápida, barata com bons resultados associados ao método de aferição indireta da PA em mm/Hg (ANDRADE et al., 2015; LOBO et al., 2017).

A média dos valores pressóricos encontrados na população de estudo foram PAS $=122 \pm 18 \mathrm{mmHg}$ e PAD $=71 \pm 11 \mathrm{mmHg}$. Quando analisamos os valores pressóricos da populaçáo autorreferida hipertensa e não hipertensa, encontramos, respectivamente, os seguintes valores: $\mathrm{PA}=125 \times 73 \mathrm{mmHg}$ e $\mathrm{PA}=120 \times 69 \mathrm{mmHg}$. De acordo com a VII Diretriz Brasileira de Hipertensão, os indivíduos considerados hipertensos encontravam-se com a pressão arterial 
controlada. Paralelamente a este achado, a população "não hipertensa" foi considerada normotensa. Esses achados sugerem a participação ativa dos indivíduos no controle da doença hipertensiva. Ademais, constatamos uma adesão de $82,4 \%$ da população à prática regular de atividade física, contribuindo para esses achados. Ensaio clínico randomizado demonstrou que a média dos valores pressóricos dos participantes apresentou diminuição dos valores de PA quando submetidos a treinamento físico associado a orientaçóes em saúde e nutricionais (RADOVANOVIC et al., 2016).

Apesar da boa adesão às práticas de atividade física, observamos o IMC classificado como excesso de peso. Além disso, 75\% dos indivíduos referiam que receberam tratamento ou orientação sobre o Diabetes Mellitus tipo 2. Estudos têm revelado que a resistência à insulina relaciona-se com maiores valores de IMC que, por conseguinte, está relacionada com a HAS (MACHADO et al., 2016; CARVALHO et al., 2015; DANIEL et al., 2013).

Em relaçáo ao histórico familiar de doença cardiovascular, observamos a prevalência de $51,5 \%$ na população do estudo. Esses achados corroboram com os dados de estudos que mostram a alta prevalência dos indivíduos em possuir um ou mais familiares com doenças cardiovasculares (DANIEL et al., 2013).

Todos os indivíduos receberam orientaçóes por meio do diálogo e entrega de fôlderes. Ressalta-se que 66,2\% receberam orientaçóes quanto aos valores limítrofes da PA, 82,4\% sobre educação direcionada à dieta saudável e $76,5 \%$ dos participantes receberam orientaçôes quanto à prática de atividade física. Sabe-se que baixos níveis de conhecimento sobre a doença estão associados a um pior controle dos níveis pressóricos, portanto, conhecer a própria doença, cuidados preventivos, troca de experiência, bem como o diálogo entre outras metodologias eficazes são essenciais para que pacientes com HAS possam exercer um autocuidado mais efetivo (TEIXEIRA et al., 2016).

Estudos têm comprovado que a aplicação de estratégias de educação em saúde participativa é um importante instrumento para estimular mudanças no estilo de vida e reduzir eventos adversos cardiovasculares. Como resultado, tem se observado a redução da pressão arterial, a diminuição do peso corporal e da circunferência da cintura, melhora 
do perfil lipídico e da glicose sanguínea, mudanças favoráveis no consumo alimentar habitual e aumento do conhecimento sobre o processo saúde-doença-cuidado (MACHADO et al., 2016).

As limitaçóes encontradas no presente estudo são decorrentes da população e local do estudo, sendo um local propício às práticas de atividades físicas, no qual se pode inferir que a população seja adepta a esses hábitos de estilo de vida.

\section{CONSIDERAÇÕES FINAIS}

Observamos que os valores pressóricos da população do estudo encontraram-se controlados e normotensos, respectivamente, tanto para os indivíduos autorreferidos hipertensos quanto para os indivíduos autorreferidos não hipertensos.

Essa pesquisa articulada com a extensão nos permitiu um olhar crítico e holístico perante os processos de saúde e doença na comunidade estudada, além de proporcionar aos pesquisadores a criação de parcerias entre projetos que apoiem o incentivo do rastreamento da pressão arterial na população, prevenindo futuros agravos e complicaçóes.

Entendemos a necessidade de maiores estudos de rastreamento de pressão arterial e identificação da prevalência de HAS na população em geral, sendo essa uma condição essencial para possíveis programas de educação em saúde nas comunidades estudadas.

\section{REFERÊNCIA}

ANDRADE, S. S. de A. et al. Prevalência de hipertensão arterial autorreferida na população brasileira: análise da Pesquisa Nacional de Saúde, 2013. Epidemiologia e Serviços de Saúde, Brasília, v. 24, n. 2, p. 297-304, jun. 2015. doi: http://dx.doi.org/10.5123/S167949742015000200012 .

ARRUDA, G. O. et al. Association between self-reported health and sociodemographic characteristics with cardiovascular diseases in adults. Revista da Escola de Enfermagem da Usp, São Paulo, v. 49, n. 1, p.61-68, fev. 2015. Doi: http://dx.doi.org/10.1590/S0080- 
623420150000100008.

BEEVERS, G.; LIP, G. Y. H.; O’BRIEN, E. ABC of hypertension: blood pressure measurement: Part II - Conventional sphygmomanometry: technique of auscultatory blood pressure measurement. BMJ: British Medical Journal, v. 322, n. 7.293, p. 1.043-1.047, April 2001.

BRASIL. Ministério da Saúde. Departamento de Informática do Sistema Único de Saúde (DATASUS). Brasília. Disponível em: $<$ http://tabnet.datasus.gov.br/cgi/tabcgi.exe?hiperdia/cnv/hdmg.def>. Acesso em: 20 jun. 2018.

CARVAlHO, C. A. et al. Associação entre fatores de risco cardiovascular e indicadores antropométricos de obesidade em universitários de São Luís, Maranhão, Brasil. Ciência \& Saúde Coletiva, Rio de Janeiro, v. 20, n. 2, p.479-490, fev. 2015. doi: http:// dx.doi.org/10.1590/1413-81232015202.02342014.

DANIEL, A. C. Q. G; VEIGA, E. V. Fatores que interferem na adesão terapêutica medicamentosa em hipertensos. Einstein, Ribeirão Preto, v. 11, n. 3, p. 331-337, set. 2013. doi: http://dx.doi.org/10.1590/ S1679-45082013000300012.

ESTACIO, R. O. et al. Effect of blood pressure control on diabetic microvascular complications in patients with hypertension and type 2 diabetes. Diabetes care, v. 23, n. 2, p. 54-64, 2000. doi: 10.1046/j.1523-1755.2002.00213.x.

FERES, F. et al. Diretriz da sociedade brasileira de cardiologia e da sociedade brasileira de hemodinâmica e cardiologia intervencionista sobre intervençáo coronária percutânea. Arquivos Brasileiros de Cardiologia, v. 109, n. 1, p. 1-81, 2017. doi: 10.5935/abc.20170111.

JARDIM, P. C. B. V. et al. I RBH - First Brazilian Hypertension Registry. Arquivos Brasileiros de Cardiologia, São Paulo, v. 10, n. 2, p. 93-98, 2016. doi: http://dx.doi.org/10.5935/abc.20160120.

LOBO, L. A. C. et al. Tendência temporal da prevalência de hipertensão arterial sistêmica no Brasil. Cadernos de Saúde Pública, Rio de Janeiro, v. 33, n. 6, p.1-13, jul. 2017. doi: http://dx.doi. org/10.1590/0102-311x00035316.

MACHADO, J. C. et al. Análise de três estratégias de educação em 
saúde para portadores de hipertensão arterial. Ciência \& Saúde Coletiva, Rio de Janeiro, v. 21, n. 2, p. 611-620, fev. 2016. doi: http://dx.doi.org/10.1590/1413-81232015212.20112014.

MINISTÉRIO DA SAÚDE. Caderno de Atenção Primária. n. 29, Brasília: MS, 2010.

PASSOS, V. M. de A.; ASSIS, T. D.; BARRETO, S. M. Hipertensão arterial no Brasil: estimativa de prevalência a partir de estudos de base populacional. Epidemiologia e serviços de Saúde, Brasília, v. 15, n. 1, p. 35-45, 2006. doi: http://dx.doi.org/10.5123/S167949742006000100003.

RADOVANOVIC, C. A. T. et al. Intervenção multiprofissional em adultos com hipertensão arterial: ensaio clínico randomizado. Revista Brasileira de Enfermagem, Brasília, v. 69, n. 6, p. 1.067-1.073, 2016. doi: http://dx.doi.org/10.1590/0034-7167-2016-0320.

SCHRIER, R. W. et al. Effects of aggressive blood pressure control in normotensive type 2 diabetic patients on albuminuria, retinopathy and strokes. Kidney international, v. 61, n. 3, p. 1.086-1.097, 2002. Doi: 10.1046/j.1523-1755.2002.00213.x

TEIXEIRA, J. de F. et al. Hypertensives' knowledge about high-sodium foods and their behavior. Arquivos Brasileiros de Cardiologia, São Paulo, v. 106, n. 5, p. 404-410, 2016. doi: http://dx.doi.org/10.5935/ abc. 20160049 .

TOlEDO, M. M.; RODRIGUES, S. de C.; CHIESA, A. M. Educação em saúde no enfrentamento da hipertensão arterial: uma nova ótica para um velho problema. Texto Contexto Enfermagem, Florianópolis, v. 16, n. 2, p. 233-8, 2007. doi: http://dx.doi. org/10.1590/S0104-07072007000200004.

UNIVERSIDADE FEDERAL DE UBERLÂNDIA. Resolução no 04/2009 do Conselho Universitário. Disponível em: <http://www. reitoria.ufu.br/Resolucoes/ataCONSUN-2009-4.pdf>. Acesso em: 9 jul. 2018.

Submetido em 12 de julho de 2018. Aprovado em 21 de agosto de 2018. 